\title{
Associations among depression severity, painful physical symptoms, and social and occupational functioning impairment in patients with major depressive disorder: a 3-month, prospective, observational study
}

This article was published in the following Dove Press journal:

Neuropsychiatric Disease and Treatment

19 September 2017

Number of times this article has been viewed

\section{Eiji Harada' \\ Yoichi Satoi ${ }^{2}$ \\ Atsushi Kuga' \\ Hirofumi Tokuoka' \\ Toshiaki Kikuchi ${ }^{3}$ \\ Koichiro Watanabe ${ }^{4}$ \\ Levent Alev' \\ Masaru Mimura ${ }^{3}$}

'Biomedicine, Medicines Development Unit Japan, Eli Lilly Japan K.K, Kobe, Japan; ${ }^{2}$ Statistical Science, Eli Lilly Japan K.K., Kobe, Japan; ${ }^{3}$ Department of Neuropsychiatry, Keio University School of Medicine, Tokyo, Japan; ${ }^{4}$ Department of Neuropsychiatry, Kyorin University School of Medicine, Tokyo, Japan
Correspondence: Hirofumi Tokuoka Biomedicine, Medicines Development Unit Japan, Eli Lilly Japan K.K., 7-I-5 Isogamidori, Chuo-Ku, Kobe, 65I-0086, Japan

Tel +8I 355749234

Fax +8I 355749979

Email tokuoka_hirofumi@lilly.com
Purpose: To investigate associations among depression severity, painful physical symptoms (PPS), and social and occupational functioning impairment in patients with major depressive disorder (MDD) who had achieved complete remission (CR) or partial remission (PR) after acute treatment.

Patients and methods: This was a 12-week, multicenter, prospective, observational study. Patients with MDD treated with an antidepressant medication for the previous 12 weeks ( \pm 3 weeks) who had achieved CR (defined as a 17-item Hamilton Rating Scale for Depression [HAM-D17] score $\leq 7$ ) or PR (HAM-D17 score $\geq 8$ and $\leq 18$ ) were enrolled. Depression severity, PPS, and impairment in social and occupational functioning were assessed using the HAM-D17, the Brief Pain Inventory (Short Form) (BPI-SF), and the Social and Occupational Functioning Assessment Scale (SOFAS), respectively, at enrollment (Week 12) and after 12 weeks (Week 24).

Results: Overall, 323 Japanese patients with MDD were enrolled (CR n=158, PR n=165) and 288 patients completed the study (CR n=139, PR n=149). HAM-D17 and SOFAS scores were strongly and negatively correlated at enrollment (Week 12; $P<0.0001)$ and Week $24(P<0.0001)$. A weak negative correlation between the BPI-SF and SOFAS was observed at Week 24 ( $P=0.0011)$, but not at enrollment $(P=0.164)$. Remission status at enrollment (CR or PR) was associated with achieving normal social and occupational functioning (SOFAS score $\geq 80$ ) at Week 24 in patients who had not achieved normal social and occupational functioning (SOFAS score $<80$ ) at enrollment (CR vs $\mathrm{PR}, \mathrm{OR}=0.05$ [95\% CIs 0.01-0.18], $P<0.0001$ ). A greater proportion of patients with CR and no PPS at enrollment achieved SOFAS scores $\geq 80$ at Week 24 than those with CR and PPS.

Conclusion: Our results suggest that treating both depressive symptoms and PPS is important for achieving a normal level of functioning on a long-term basis in patients with MDD.

Keywords: depression, major depressive disorder, painful physical symptoms, remission, social/occupational functioning

\section{Introduction}

The optimal treatment outcome in major depressive disorder (MDD) is remission, a period when the affected individual becomes asymptomatic - that is, no longer meets syndromal criteria for MDD and has no more than minimal symptoms. ${ }^{1,2}$ 
However, despite recent advances in the pharmacological treatment of MDD, a substantial proportion of individuals fail to achieve remission. Indeed, approximately one third of patients experience persistent residual symptoms and fail to reach remission. ${ }^{3,4}$ A previous study reported that patients with MDD who had residual symptoms relapsed more than three times faster than those who did not have residual symptoms. ${ }^{5}$

The classification of MDD has focused primarily on emotional symptoms (eg, depressed mood, reduced interest/ pleasure, feelings of worthlessness, and excessive guilt). However, painful physical symptoms (PPS) are also known to emerge during the course of depression. ${ }^{6}$ Studies have shown that approximately half of patients with mild to moderate depression may also experience PPS ${ }^{7}$ and that the severity of PPS is higher in patients with MDD than in healthy controls. ${ }^{8}$ Moreover, general somatic symptoms, which include tiredness, loss of energy, fatigue, and muscular aches and pain, are some of the most frequent residual symptoms of MDD. ${ }^{9}$

Some studies suggest that persistence of residual symptoms, in general, is associated with functional impairment, ${ }^{10}$ and that the presence of residual PPS is negatively associated with social and occupational functioning at the end of acute treatment (12 weeks). ${ }^{11,12}$ However, it is not understood how improvement in social and occupational functioning is associated with depression severity and PPS in the long term (ie, beyond acute treatment). Notably, how residual PPS (after achieving remission) affects subsequent recovery of social and occupational functioning requires clarification.

We recently conducted a prospective, observational study of outpatients with MDD to assess the patterns of PPS and emotional symptoms among patients with partial remission (PR) or complete remission (CR) of an MDD episode. ${ }^{13}$ Findings from this study showed that PPS was one of the commonly observed residual symptoms, together with core emotional symptoms (eg, loss of interest, depressed mood), that PPS was associated with PR as defined by 17-item Hamilton Rating Scale for Depression (HAM-D17) score, and that PR was associated with a higher prevalence of at least moderate PPS in patients with MDD who had been treated with antidepressant medication for 12 weeks. Here, we report findings from a subsequent follow-up study, conducted for a secondary objective, in which we investigated associations among PPS, depression severity, and impairment in social and occupational functioning in patients with MDD who had achieved CR or PR after 12 weeks of acute treatment with antidepressant medication. We aimed to assess how residual PPS affects subsequent recovery, hypothesizing that residual PPS is negatively associated with improvements in depression symptoms and in social and occupational functioning.

\section{Materials and methods Study design}

This was a 12-week extension of a multicenter, prospective, observational study of patients with MDD treated at 27 psychiatric and psychosomatic outpatient clinics in Japan. ${ }^{13}$ Patients with PR or CR after 12 weeks of treatment were enrolled and pair-wise matched by sex (two strata: male and female) and age (three strata: $20-39,40-65$, and $\geq 66$ years) to ensure equal numbers of patients with PR and $\mathrm{CR}$ in each sex and age stratum. Patients were assessed at enrollment (ie, Week 12 in the primary study) and after 12 weeks (Week 24; Figure S1).

The study protocol was reviewed and approved by participating institutional ethics review boards when applicable, and the study was conducted in accordance with the laws and regulations governing clinical research in Japan, as appropriate. All patients provided written informed consent before participating in the study.

\section{Study population}

The study population has been described in detail previously. ${ }^{13}$ In brief, outpatients were enrolled if they were aged $\geq 20$ years, had MDD without psychotic features (as defined by the Diagnostic and Statistical Manual of Mental Disorders, Fourth Edition, Text Revision), ${ }^{6}$ were treated for an MDD episode with antidepressant medication for the previous $12( \pm 3)$ weeks, and were in CR (defined as a HAM-D17 score $\leq 7)^{14}$ or PR (HAM-D17 score $\geq 8$ and $\leq 18$ ). ${ }^{9}$ The physician and patient had sole discretion on the antidepressant medication regimen (ie, type and dose) prescribed, according to the usual standard of care.

Exclusion criteria were a previous diagnosis of bipolar disorder, schizophrenia or other psychotic disorder, a current diagnosis of dysthymic disorder or adjustment disorder, or a HAM-D17 score $\geq 19$. $^{13}$

\section{Assessments}

All assessments were conducted by patients and their physicians. Depression severity, PPS, and social and occupational functioning impairment were assessed at enrollment (Week 12) and after 12 weeks (Week 24).

Depression severity was assessed using the HAM-D17, a 17-item, clinician-rated scale,,$^{9,15}$ in conjunction with the Structured Interview Guide for the HAM-D17. ${ }^{16-18}$ 
PPS and severity were assessed using the Brief Pain Inventory (Short Form) (BPI-SF). The BPI-SF is a 9-item, self-assessment tool completed by the patient, who evaluates pain severity and its interference with activities of daily living using a 0 (no pain/does not interfere) to 10 (pain as severe as you can imagine/completely interferes) scale. ${ }^{19,20}$ Patients completed item 1, which identifies the presence of pain of any severity. Patients who had any pain were defined as PPS positive (PPS+) and completed items 2 (identifies the body site of pain) through to 6 (assesses the intensity of worst pain [item 3], least pain [item 4], and average pain [item 5] over the last 24 hours, and current pain [item 6]); item 8 (pain relief by current treatments or medications), and item 9 (assesses the degree to which pain interferes with daily activities). Information regarding the types of pain relief treatments and medications (item 7) was not collected. Patients who had no pain (item $1=0$ ) were defined as PPS negative (PPS-) and were assigned scores of 0 for items 3 to 6,8 , and 9 .

Social and occupational functioning impairment was assessed using the Social and Occupational Functioning Assessment Scale (SOFAS), a 100-point, single-item scale that rates functioning across a continuum from severely impaired (1) to optimal (100). ${ }^{21}$ Functioning was considered normal if a SOFAS score was $\geq 80 .{ }^{10}$

\section{Statistical analysis}

The investigators or appointed personnel entered data into an electronic data collection application and investigators validated the data for correctness with a written signature.

Patient sociodemographic and clinical characteristics at enrollment (Week 12), and HAM-D17, BPI-SF average pain (item 5), and SOFAS scores at enrollment (Week 12) and Week 24 were summarized for patients with CR and PR using descriptive statistics; continuous data are presented as number of observations (n), mean, and SD, and categorical data are presented as number of patients providing data at the relevant time point (n) and percentages.

Pearson correlation coefficients $(r)$ were calculated between HAM-D17 and BPI-SF average pain (item 5) scores, HAM-D17 and SOFAS scores, and BPI-SF average pain (item 5) and SOFAS scores at enrollment (Week 12) and at Week 24. The BPI-SF 24-hour average pain score was used in these analyses because it was the primary outcome measure for pain in studies of $\mathrm{MDD}^{22-26}$ and its use is endorsed by the IMMPACT recommendations for clinical pain trials. ${ }^{27-29}$

Factors associated with a SOFAS score $\geq 80$ at Week 24 (response variable) in patients with a SOFAS score $<80$ at enrollment (Week 12) were investigated using a logistic regression model by stepwise methods. Explanatory variables were the following factors at enrollment (Week 12): sex, age, age at first depressive episode, number of previous depressive episodes, duration of current MDD episode, previous diagnosis of personality disorders, alcohol intake condition, presence of physical comorbidities that may cause PPS, hospitalization, early retirement, sick leave, class of antidepressant medication (selective serotonin reuptake inhibitor [SSRI], serotonin norepinephrine reuptake inhibitor [SNRI], tricyclic, tetracyclic, others), analgesic treatment (no, yes) and class (non-steroidal anti-inflammatories, non-opioid), benzodiazepine treatment (no, yes) and purpose of use (insomnia, anxiety, other), specific psychotherapy, presence of PPS (BPI-SF item 1), body site of pain (BPI-SF item 2), worst pain (BPI-SF item 3), least pain (BPI-SF item 4), average pain (BPI-SF item 5), current pain (BPI-SF item 6), pain relief by current treatments or medications (BPI-SF item 8), interference of pain with daily activities (BPI-SF item 9), remission status (CR or PR at enrollment), and depression improvement (HAM-D17 total score change from enrollment [Week 12] to Week 24). The threshold for explanatory variable selection was $P=0.15$.

The proportions of patients with CR and PPS+ or PPS- at enrollment (Week 12) who achieved a SOFAS score $\geq 80$ at enrollment (Week 12) and at Week 24 were analyzed using a chi-square test.

All statistical tests were based on a 2-sided significance level of 0.05 , except for the logistic regression analyses $(P=0.15)$. Analyses were done using SAS version 9.1 .3 or later (SAS Institute Inc., Cary, NC, USA).

\section{Results \\ Patient disposition}

A total of 325 Japanese patients with MDD consented to participate in the study. Of these patients, 323 were enrolled (CR $n=158, P R n=165)$ and 288 completed the study (CR $n=139, P R n=149)$. Of the 149 patients with PR at enrollment who completed the study, 70 (47\%) achieved CR, 73 (49\%) remained as PR, and six (4\%) had missing data by Week 24. Of the 139 patients with CR at enrollment who completed the study, 124 (89\%) remained as CR, ten (7\%) were PR, and five (4\%) had missing data by Week 24 . The most frequently reported reason for study discontinuation was failure to follow up (CR n=15 [9.5\%], PR n=11 [6.7\%]).

\section{Patient characteristics}

The sociodemographic and clinical characteristics of patients have been previously described. ${ }^{13}$ Sociodemographic 
characteristics were similar for the CR and PR groups; mean \pm SD age was $47.7 \pm 14.9$ and $44.8 \pm 14.7$ years, respectively, and the n (\%) of female patients was $80(50.6 \%)$ and 88 (53.3\%), respectively. Clinical characteristics were also similar between groups including the mean \pm SD age at onset of first depressive episode (CR 43.1 \pm 15.6 years [ $n=146]$, PR 40.0 \pm 13.7 years $[n=151])$, mean \pm SD number of previous depressive episodes (CR 1.6 \pm 1.1 [n=109], PR 1.5 \pm 0.9 $[\mathrm{n}=114])$, and mean \pm SD total duration of the current MDD episode (CR 31.4 \pm 63.9 weeks [n=158], PR 36.0 \pm 49.4 weeks $[\mathrm{n}=165])$. Most patients were treated with an SSRI (CR $\mathrm{n}=75$ [47.5\%], PR $\mathrm{n}=72$ [43.6\%]) or an SNRI (CR $\mathrm{n}=59$ [37.3\%], PR n=59 [35.8\%]) as antidepressant medication before enrollment (Week 12). Tricyclic, tetracyclic or other antidepressants were used to a lesser extent. Physical comorbidities which may have caused PPS at enrollment are shown in Table $\mathrm{S} 1$.

\section{Depression severity, PPS, and social and occupational functioning impairment at enrollment (Week I2) and Week 24 Mean HAM-DI7, BPI-SF average pain, and SOFAS scores}

Mean HAM-D17, BPI-SF average pain (item 5), and SOFAS scores for patients with $\mathrm{CR}$ and with PR are presented in Table 1. Patients with PR had a numerically higher mean HAM-D17 score than those with CR at enrollment (Week 12), as expected, and at Week 24. Patients with PR

Table I Mean scores for HAM-D I7, BPI-SF average pain (item 5), and SOFAS at enrollment and Week 24

\begin{tabular}{|c|c|c|c|c|}
\hline \multirow[t]{2}{*}{ Variable } & \multicolumn{2}{|l|}{ CR group } & \multicolumn{2}{|l|}{ PR group } \\
\hline & $\begin{array}{l}\text { Enrollment } \\
\text { (Week I2) }\end{array}$ & Week 24 & $\begin{array}{l}\text { Enrollment } \\
\text { (Week I2) }\end{array}$ & Week 24 \\
\hline \multicolumn{5}{|l|}{ HAM-DI7 } \\
\hline $\begin{array}{l}\text { Number of } \\
\text { patients }\end{array}$ & 158 & 136 & 165 & 146 \\
\hline Mean \pm SD & $4.4 \pm 2.0$ & $3.5 \pm 3.0$ & $11.8 \pm 3.1$ & $8.7 \pm 5.2$ \\
\hline \multicolumn{5}{|c|}{ BPI-SF average pain (item 5) } \\
\hline $\begin{array}{l}\text { Number of } \\
\text { patients }\end{array}$ & 158 & 134 & 165 & 143 \\
\hline Mean \pm SD & $0.9 \pm 1.5$ & $0.8 \pm I .4$ & $1.7 \pm 2.0$ & $1.4 \pm 2.0$ \\
\hline \multicolumn{5}{|l|}{ SOFAS } \\
\hline $\begin{array}{l}\text { Number of } \\
\text { patients }\end{array}$ & 145 & 126 & 153 & 138 \\
\hline Mean \pm SD & $75.5 \pm 10.7$ & $78.2 \pm 11.5$ & $58.9 \pm 11.3$ & $64.7 \pm 14.4$ \\
\hline
\end{tabular}

Note: aPatients who answered "No" to BPI-SF (item I) were allocated a score of 0 for the analyses of BPI-SF average pain (item 5).

Abbreviations: BPI-SF, Brief Pain Inventory (Short Form); CR, complete remission; HAM-D 17, 17-item Hamilton Rating Scale for Depression; PR, partial remission; SOFAS, Social and Occupational Functioning Assessment Scale. also had a numerically higher mean BPI-SF average pain score than those with CR at both time points. Patients with PR had a numerically lower mean SOFAS score than those with CR both at enrollment (Week 12) and at Week 24. In summary, patients with PR presented with greater depression severity, PPS, and social and occupational functioning impairment than those with CR at enrollment (Week 12) and at Week 24.

\section{Correlations between HAM-D I7, BPI-SF average pain, and SOFAS scores}

Correlations between HAM-D17, BPI-SF average pain (item 5), and SOFAS scores are presented in Table 2. HAM-D17 and SOFAS scores were strongly and negatively associated at both enrollment (Week 12) and at Week 24 (both $P<0.0001$ ). No association between BPI-SF average pain and SOFAS scores was found at enrollment (Week 12), whereas a weak negative association was found at Week 24 $(P=0.0011)$. HAM-D17 and BPI-SF average pain scores were found to be positively associated, with a weak association at enrollment (Week 12) and a moderate association at Week 24 (both $P<0.0001$ ). In summary, increased social and occupational functioning was found to be associated with reduced depression severity at enrollment (Week 12) and Week 24, and with reduced PPS at Week 24.

\section{Variables associated with normal social and occupational functioning (SOFAS score $\geq 80$ ) at Week 24}

To identify variables associated with achieving normal social and occupational functioning, a stepwise logistic regression analysis was done for patients with a SOFAS

Table 2 Correlations among HAM-DI7, BPI-SF average pain (item 5), and SOFAS scores

\begin{tabular}{|c|c|c|c|}
\hline $\begin{array}{l}\text { Time point for } \\
\text { evaluation }\end{array}$ & $\mathbf{n}$ & $r$ & $P$-value \\
\hline \multicolumn{4}{|c|}{ HAM-DI7 and BPI-SF average pain (item 5) } \\
\hline Enrollment (Week 12) & 323 & 0.31 & $<0.000$ I \\
\hline Week 24 & 276 & 0.44 & $<0.000$ I \\
\hline \multicolumn{4}{|l|}{ HAM-DI7 and SOFAS } \\
\hline Enrollment (Week 12) & 298 & -0.65 & $<0.000$ I \\
\hline Week 24 & 263 & -0.63 & $<0.0001$ \\
\hline \multicolumn{4}{|c|}{ BPI-SF average pain (item 5$)^{a}$ and SOFAS } \\
\hline Enrollment (Week 12) & 298 & -0.08 & 0.164 \\
\hline Week 24 & 264 & -0.20 & 0.0011 \\
\hline
\end{tabular}

Note: aPatients who answered "No" to BPI-SF (item I) were allocated a score of 0 for the analyses of BPI-SF average pain (item 5).

Abbreviations: BPI-SF, Brief Pain Inventory (Short Form); HAM-DI7, 17-item Hamilton Rating Scale for Depression; SOFAS, Social and Occupational Functioning Assessment Scale. 
Table 3 ORs associated with achieving a SOFAS score $\geq 80$ at Week 24 in patients with a SOFAS score $<80$ at enrollment (Week I2)

\begin{tabular}{|c|c|c|c|}
\hline $\begin{array}{l}\text { Explanatory variables } \\
\text { (at enrollment [Week I2]) }\end{array}$ & OR & $95 \% \mathrm{Cl}$ & $P$-value \\
\hline Remission status (CR: 0, PR: I) & 0.05 & $0.01-0.18$ & $<0.0001$ \\
\hline $\begin{array}{l}\text { BPI-SF worst pain (item 3) } \\
\text { (continuous variable) }\end{array}$ & 0.46 & $0.25-0.83$ & 0.0098 \\
\hline $\begin{array}{l}\text { BPI-SF least pain (item 4) } \\
\text { (continuous variable) }\end{array}$ & 2.94 & $0.98-8.88$ & 0.0553 \\
\hline $\begin{array}{l}\text { Presence of physical comorbidities } \\
\text { that may cause PPS (No: } 0 \text {, Yes: I) }\end{array}$ & 0.16 & $0.02-1.02$ & 0.0522 \\
\hline Class of antidepressant medication & & & \\
\hline SSRI (No: 0, Yes: I) & 5.44 & $|| 2-26.4 \mid$. & 0.0356 \\
\hline SNRI (No: 0, Yes: I) & 11.60 & $1.97-68.14$ & 0.0067 \\
\hline $\begin{array}{l}\text { Benzodiazepine agonist treatment and } \\
\text { purpose of use (No: } 0 \text {, Anxiety: I) }\end{array}$ & 0.40 & $0.14-1.14$ & 0.0861 \\
\hline $\begin{array}{l}\text { Depression improvement }{ }^{\mathrm{b}} \\
\text { (continuous variable) }\end{array}$ & 0.82 & $0.69-0.96$ & 0.0132 \\
\hline
\end{tabular}

Notes: The analysis was based on 140 patients who had a SOFAS score $<80$ at enrollment and a recorded SOFAS score at Week 24 , and had no missing data for the explanatory variables at enrollment. Logistic regression analysis by stepwise methods was used. "Patients who answered "No" to BPI-SF (item I) were allocated a score of 0 for the analyses of BPI-SF worst pain (item 3) and BPI-SF least pain (item 4). 'Depression improvement was the change in HAM-DI7 total score from enrollment (Week 12) to Week 24.

Abbreviations: BPI-SF, Brief Pain Inventory (Short Form); CR, complete remission HAM-DI7, I7-item Hamilton Rating Scale for Depression; PPS, painful physical symptoms; PR, partial remission; SNRI, serotonin norepinephrine reuptake inhibitor; SOFAS, Social and Occupational Functioning Assessment Scale; SSRI, selective serotonin reuptake inhibitor.

score $<80$ at enrollment (Week 12; CR n=83, PR n=148; Table 3 ). Of note, patients with CR were significantly more likely to achieve SOFAS score $\geq 80$ at Week 24 than those with PR $(P<0.0001)$. Other explanatory variables significantly associated with achieving a SOFAS score $\geq 80$ at Week 24 were BPI-SF worst pain (item 3) $(P=0.0098)$, the class of antidepressant medication (SSRI, $P=0.0356$; SNRI, $P=0.0067)$, and depression improvement $(P=0.0132)$.

\section{Effect of residual PPS at enrollment (Week 12) on achieving normal social and occupational functioning (SOFAS score $\geq 80$ ) at Week 24 in patients with $C R$}

The effect of residual PPS on functional recovery in patients with CR was assessed using the percentage of patients with a SOFAS score $\geq 80$ at enrollment (Week 12) and at Week 24 (Figure 1). The proportion of patients with CR and PPS- at enrollment who had a SOFAS score $\geq 80$ increased from $42.2 \%$ at enrollment (Week 12) to $62.6 \%$ at Week 24 . In contrast, there was very little change between enrollment and Week 24 in the proportion of patients with

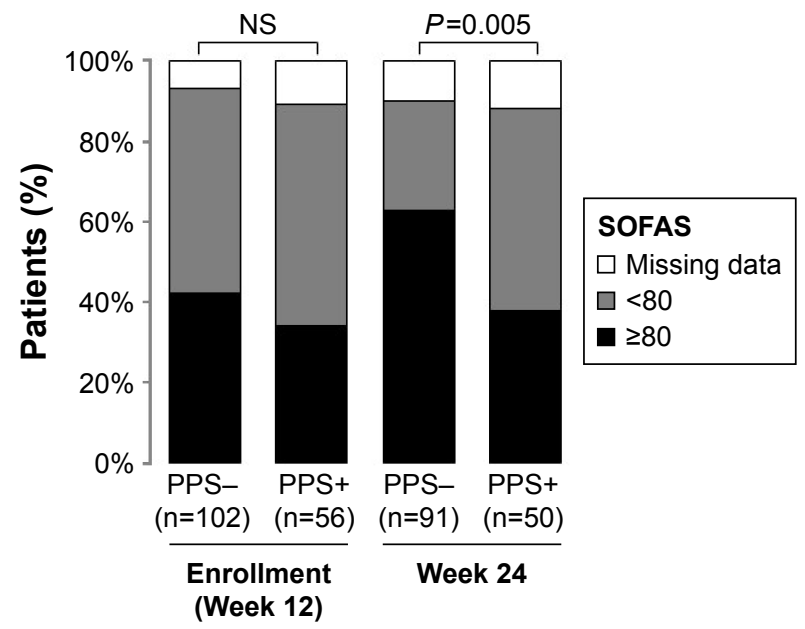

Figure I The proportion of patients with CR and PPS- or PPS+ at enrollment (Week 12) who had SOFAS scores $<80$ or $\geq 80$ at enrollment (Week 12) and Week 24.

Notes: "Missing data" indicates patients whose case report forms were collected, but on whom the SOFAS assessment was not performed. $P=0.005$, chi-square test. Abbreviations: CR, complete remission; NS, not significant; PPS-, painful physical symptoms negative; PPS+, painful physical symptoms positive; SOFAS, Social and Occupational Functioning Assessment Scale.

CR and PPS+ at enrollment who had a SOFAS score $\geq 80$ (33.9\% and $38.0 \%$, respectively). A significantly greater proportion of patients with PPS- than those with PPS+ had a SOFAS score $\geq 80$ by Week $24(P=0.005)$. In summary, residual PPS at Week 12 had a negative effect on achieving normal social and occupational functioning, even after CR of depressive symptoms.

\section{Discussion}

The results of our study provide evidence that residual PPS is associated with impairment in social and occupational functioning 6 months after treatment initiation. In addition, our results suggest that residual PPS hinders the recovery of social and occupational functioning in patients, even if they have achieved remission. These results highlight the importance of treating both depressive symptoms and PPS to achieve normal social and occupational functioning on a long-term basis.

To our knowledge, this is the first study to explore the association between PPS severity and social and occupational functional impairment in a longitudinal manner after acute treatment for MDD. Our results indicated weak to strong correlations between scores from the three assessment scales (HAMD17, BPI-SF average pain, and SOFAS), except for SOFAS and BPI-SF average pain scores at enrollment (Week 12). The strong negative correlation between HAM-D17 and SOFAS scores suggests that resolution of depression is closely associated with recovery of social and occupational functioning, 
whereas the weak negative correlation between SOFAS and BPI-SF average pain scores at Week 24 suggests that the association between improved PPS and recovery of social and occupational functioning may develop afterwards. Although the correlation analyses do not prove a causal relationship, they suggest that it may be beneficial to continue to monitor PPS, even after CR of depressive symptoms.

We further investigated what factors at enrollment (Week 12) would predict achieving a SOFAS score $\geq 80$ at Week 24 in patients with a SOFAS score $<80$ at enrollment (Week 12) (Table 3). Our results indicate that a patient's remission status after 12 weeks of acute antidepressant treatment was strongly associated with achieving normal social and occupational functioning after another 12 weeks of antidepressant treatment. This finding confirms the importance of attaining $\mathrm{CR}$ by treating the wide range of depressive symptoms. In addition, the degree of BPI-SF worst pain (item 3) was significantly associated with achieving a SOFAS score $\geq 80$. Although the degree of BPI-SF average pain (item 5) was not a significant predictor for achieving a SOFAS score $\geq 80$, the results of the logistic regression analysis suggest that the presence of PPS can have a negative influence on the subsequent recovery of social and occupational functioning. Thus, in addition to other identified variables, such as depression improvement (HAM-D17 total score change from enrollment [Week 12] to Week 24), the presence of PPS is a negative contributor to social and occupational functioning and should be monitored, even after the acute treatment phase and resolution of an MDD episode. Although the class of antidepressant medication was also identified as a significant factor, the CI for this association was large. Therefore, it is not possible to draw any conclusions about the effectiveness of specific types of antidepressants.

Findings from our study suggest that the resolution of depressive symptoms and PPS during the acute phase of treatment is important for achieving normal social and occupational functioning. A significantly higher percentage of patients with CR and PPS- had normal social and occupational functioning by Week 24 than those with CR and PPS+. Thus, although achieving CR is critical, residual PPS after CR may hinder patients from recovering normal social and occupational functioning.

Findings from our study reinforce and advance our understanding of the nature of residual PPS. Romera et al ${ }^{10}$ clarified that the presence of residual symptoms, in general, was associated with functional impairment that persisted beyond the acute treatment phase; however, they did not specifically assess the presence of residual PPS. Our finding that having residual symptoms or having PR was associated with functional impairment reinforced the findings reported by Romera et al. ${ }^{10}$ In addition, our findings shed new light on how specific residual symptoms (ie, PPS) affect functional impairment. The presence of residual PPS has been previously reported to be associated with functional impairment following the acute treatment phase (12 weeks). ${ }^{11,12}$ Collectively, our findings not only confirm the critical importance of achieving CR, but also highlight the relevance of improving PPS for functional recovery.

There are several limitations with this study. First, the observational nature of this study means that only associations, rather than causal relationships, can be shown among depression severity, PPS, and social and occupational functioning impairment. Second, patients had already been treated for 12 weeks at enrollment and no information about the treatment regimens of these patients (eg, doses, duration, combinations), other than class of antidepressant medication, depression severity, PPS, and social and occupational functioning at treatment initiation, was collected. Therefore, we were unable to match patients with PR and CR according to the severity of their depressive symptoms and PPS at treatment initiation. Third, only patients who continued treatment for 12 weeks (and achieved PR or CR before enrollment) were recruited for the study. Therefore, patients whose treatment had stopped, for whatever reason, were not included in this study. Fourth, the BPI-SF average pain score which we used to assess residual PPS only measures pain experienced in the previous 24 hours, and entire residual PPS that patients experience may not have been fully evaluated. However, the BPI-SF 24-hour average pain score is widely used for pain evaluation in studies of $\mathrm{MDD}^{22-26}$ and its use is endorsed by the IMMPACT recommendations for clinical pain trials. ${ }^{27-29}$ In addition, we did not collect information about the types of treatments and medications used for pain relief at enrollment or during the study. Last, a small minority of patients $(9.9 \%)$ had a physical comorbidity that may have caused PPS.

\section{Conclusion}

The ultimate treatment goal of MDD is the resolution of all symptoms and the full recovery of social and occupational functioning. This longitudinal study sheds light on associations among depressive symptoms, PPS, and social and occupational functioning impairment. Our results demonstrated that the presence of residual PPS may hinder patients from recovering normal social and occupational functioning levels even after achieving remission, highlighting the 
importance of treating both depressive symptoms and PPS in patients with MDD.

\section{Acknowledgments}

The authors are deeply grateful to the primary investigators, sub-investigators, and staff at the 27 study sites, and all the patients who participated in this study. Medical writing assistance was provided by Hirofumi Yamaguchi, PhD, of CMIC Co., Ltd., funded by Eli Lilly Japan K.K. Additional medical writing assistance was provided by Julie Monk, $\mathrm{PhD}, \mathrm{CMPP}$ and Serina Stretton, PhD, CMPP of ProScribe - Envision Pharma Group, and was funded by Eli Lilly Japan K.K. This study was sponsored by Eli Lilly Japan K.K. and conducted by CMIC Co., Ltd. under contract to Eli Lilly Japan K.K.

\section{Disclosure}

EH contributed to this work as a former full-time employee of Eli Lilly Japan K.K. The opinions expressed in this work are solely his and do not represent those of his current affiliation, the Japanese Ministry of Health, Labour and Welfare. YS, AK, HT, and LA are employees of Eli Lilly Japan K.K. TK contributed to this work mostly whilst employed at Keio University School of Medicine, Tokyo, Japan. The opinions expressed in this work are solely his and do not represent those of his current affiliation, the Japan Agency for Medical Research and Development (AMED). TK has received speaker fees from Astellas Pharma Inc., Sumitomo Dainippon Pharma Co., Ltd., Eli Lilly Japan K.K., GlaxoSmithKline K.K., Jansen Pharmaceutical K.K., Otsuka Pharmaceutical Co., Ltd., Pfizer Inc., and Yoshitomiyakuhin Corporation. KW has received grants from Astellas Pharma Inc., Eisai Co., Ltd., MSD K.K., Otsuka Pharmaceutical Co., Ltd., Shionogi \& Co., Ltd., Daiichi Sankyo Co., Ltd., Sumitomo Dainippon Pharma Co., Ltd., Mitsubishi Tanabe Pharma Corporation, Meiji Seika Pharma Co., Ltd., consultancy fees from Otsuka Pharmaceutical Co., Ltd., Taisho Toyama Pharmaceutical Co., Ltd., Sumitomo Dainippon Pharma Co., Ltd., Takeda Pharmaceutical Company Limited, Mitsubishi Tanabe Pharma Corporation, Eli Lilly Japan K.K., Mochida Pharmaceutical Co., Ltd., and speaker fees and payment for manuscript preparation from Astellas Pharma Inc., MSD K.K., Otsuka Pharmaceutical Co., Ltd., GlaxoSmithKline K.K., Shionogi \& Co., Ltd., Daiichi Sankyo Co., Ltd., Sumitomo Dainippon Pharma Co., Ltd., Mitsubishi Tanabe Pharma Corporation, Eli Lilly Japan K.K., Pfizer Japan Inc., Meiji Seika Pharma Co., Ltd., Janssen Pharmaceutical K.K. MM has received grants and/or speaker's honoraria from Asahi Kasei Corp., Astellas Pharma Inc., Daiichi Sankyo
Co., Ltd., Sumitomo Dainippon Pharma Co., Ltd., Eisai Co., Ltd., Eli Lilly Japan K.K., GlaxoSmithKline K.K., Janssen Pharmaceutical K.K., Meiji Seika Pharma Co., Ltd., Mochida Pharmaceutical Co., Ltd., MSD K.K., Novartis Pharma K.K., Otsuka Pharmaceutical Co., Ltd., Pfizer Inc., Shionogi \& Co., Ltd., Takeda Pharmaceutical Company Limited, Mitsubishi Tanabe Pharma Corporation, and Yoshitomiyakuhin Corporation within the past 3 years. The authors report no other conflicts of interest in this work.

\section{References}

1. Frank E, Prien RF, Jarrett RB, et al. Conceptualization and rationale for consensus definitions of terms in major depressive disorder. Remission, recovery, relapse, and recurrence. Arch Gen Psychiatry. 1991;48(9): $851-855$.

2. Keller MB. Past, present, and future directions for defining optimal treatment outcome in depression: remission and beyond. JAMA. 2003 289(23):3152-3160

3. Fava M, Davidson KG. Definition and epidemiology of treatmentresistant depression. Psychiatr Clin North Am. 1996;19(2):179-200.

4. Ramana R, Paykel ES, Cooper Z, Hayhurst H, Saxty M, Surtees PG Remission and relapse in major depression: a two-year prospective follow-up study. Psychol Med. 1995;25(6):1161-1170.

5. Judd LL, Akiskal HS, Maser JD, et al. Major depressive disorder: a prospective study of residual subthreshold depressive symptoms as predictor of rapid relapse. J Affect Disord. 1998;50(2-3):97-108.

6. American Psychiatric Association. Diagnostic and Statistical Manual of Mental Disorders (4th ed., text rev.). Washington, DC: American Psychiatric Publishing; 2000.

7. Brnabic A, Lin C, Monkul ES, Duenas H, Raskin J. Major depressive disorder severity and the frequency of painful physical symptoms: a pooled analysis of observational studies. Curr Med Res Opin. 2012;28(12): 1891-1897.

8. Kishi T, Matsuda Y, Mukai T, et al. A cross-sectional survey to investigate the prevalence of pain in Japanese patients with major depressive disorder and schizophrenia. Compr Psychiatry. 2015;59:91-97.

9. Paykel ES, Ramana R, Cooper Z, Hayhurst H, Kerr J, Barocka A. Residual symptoms after partial remission: an important outcome in depression. Psychol Med. 1995;25(6):1171-1180.

10. Romera I, Perez V, Menchon JM, Delgado-Cohen H, Polavieja P, Gilaberte I. Social and occupational functioning impairment in patients in partial versus complete remission of a major depressive disorder episode. A six-month prospective epidemiological study. Eur Psychiatry. 2010;25(1):58-65.

11. Romera I, Perez V, Ciudad A, et al. Residual symptoms and functioning in depression, does the type of residual symptom matter? A post-hoc analysis. BMC Psychiatry. 2013;13:51.

12. Romera I, Perez V, Quail D, Berggren L, Lenox-Smith A, Gilaberte I. Individual residual symptoms and functional impairment in patients with depression. Psychiatry Res. 2014;220(1-2):258-262.

13. Harada E, Satoi Y, Kikuchi T, Watanabe K, Alev L, Mimura M. Residual symptoms in patients with partial versus complete remission of a major depressive disorder episode: patterns of painful physical symptoms in depression. Neuropsychiatr Dis Treat. 2016;12:1599-1607.

14. Hamilton M. A rating scale for depression. J Neurol Neurosurg Psychiatry. 1960;23:56-62.

15. Hamilton M. Development of a rating scale for primary depressive illness. Br J Soc Clin Psychol. 1967;6(4):278-296.

16. Nakane Y, Williams JB. Structured interview guide for the Hamilton Depression Rating Scale (SIGH-D) Japanese version. Rinshō Seishin Yakuri (Japanese J Clin Pharmacol). 2003;6(10):1353-1368. Available from: http://seiwa-pb.co.jp/search/bo01/bo0103/bn/06/10index.html. Accessed August 18, 2017. Japanese. 
17. Williams JB. A structured interview guide for the Hamilton Depression Rating Scale. Arch Gen Psychiatry. 1988;45(8):742-747.

18. Williams JB. Standardizing the Hamilton Depression Rating Scale: past, present, and future. Eur Arch Psychiatry Clin Neurosci. 2001; 251 Suppl 2:II6-II12.

19. Cleeland CS, Ryan KM. Pain assessment: global use of the Brief Pain Inventory. Ann Acad Med Singapore. 1994;23(2):129-138.

20. Cleeland CS. The brief pain inventory user guide. MD Anderson Center. Available from: https://www.mdanderson.org/documents/ Departments-and-Divisions/Symptom-Research/BPI_UserGuide.pdf. Accessed August 14, 2017.

21. Goldman HH, Skodol AE, Lave TR. Revising axis V for DSM-IV: a review of measures of social functioning. Am J Psychiatry. 1992; 149(9):1148-1156.

22. Brannan SK, Mallinckrodt CH, Brown EB, Wohlreich MM, Watkin JG, Schatzberg AF. Duloxetine $60 \mathrm{mg}$ once-daily in the treatment of painful physical symptoms in patients with major depressive disorder. J Psychiatr Res. 2005;39(1):43-53.

23. Brecht S, Courtecuisse C, Debieuvre C, et al. Efficacy and safety of duloxetine $60 \mathrm{mg}$ once daily in the treatment of pain in patients with major depressive disorder and at least moderate pain of unknown etiology: a randomized controlled trial. J Clin Psychiatry. 2007;68(11): $1707-1716$
24. Gaynor PJ, Gopal M, Zheng W, et al. Duloxetine versus placebo in the treatment of major depressive disorder and associated painful physical symptoms: a replication study. Curr Med Res Opin. 2011;27(10): 1859-1867.

25. Gaynor PJ, Gopal M, Zheng W, Martinez JM, Robinson MJ, Marangell LB. A randomized placebo-controlled trial of duloxetine in patients with major depressive disorder and associated painful physical symptoms. Curr Med Res Opin. 2011;27(10):1849-1858.

26. Harada E, Tokuoka H, Fujikoshi S, et al. Is duloxetine's effect on painful physical symptoms in depression an indirect result of improvement of depressive symptoms? Pooled analyses of three randomized controlled trials. Pain. 2016;157(3):577-584.

27. Dworkin RH, Turk DC, Farrar JT, et al. Core outcome measures for chronic pain clinical trials: IMMPACT recommendations. Pain. 2005; 113(1-2):9-19.

28. Dworkin RH, Turk DC, Wyrwich KW, et al. Interpreting the clinical importance of treatment outcomes in chronic pain clinical trials: IMMPACT recommendations. J Pain. 2008;9(2):105-121.

29. Turk DC, Dworkin RH, Burke LB, et al. Developing patient-reported outcome measures for pain clinical trials: IMMPACT recommendations. Pain. 2006;125(3):208-215. 


\section{Supplementary materials}

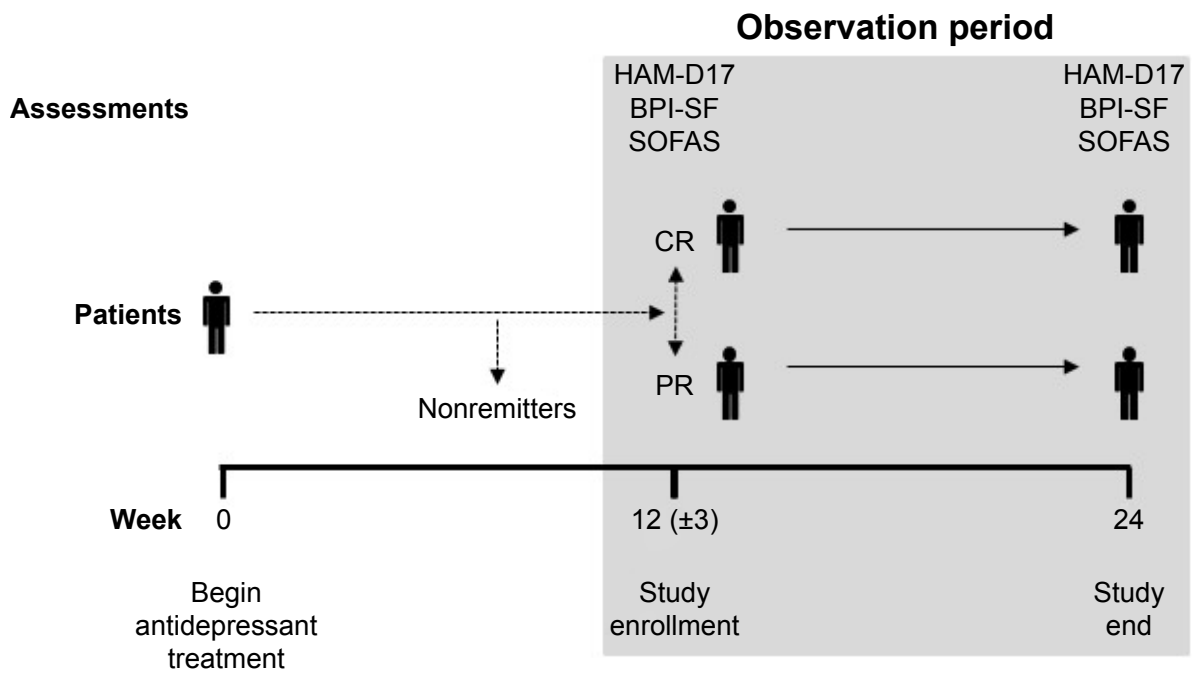

Figure SI Study design.

Abbreviations: BPI-SF, Brief Pain Inventory (Short Form); CR, complete remission; HAM-DI7, I7-item Hamilton Rating Scale for Depression; PR, partial remission; SOFAS, Social and Occupational Functioning Assessment Scale.

Table SI Physical comorbidities which may have caused PPS at enrollment

\begin{tabular}{|c|c|c|c|}
\hline CR group & $\mathbf{n}$ & PR group & $\mathbf{n}$ \\
\hline Any comorbidity & 14 & Any comorbidity & 18 \\
\hline Intervertebral disc hernia & 2 & Low back pain & 3 \\
\hline Lumbar intervertebral disc hernia & 2 & Gastritis & 2 \\
\hline Adrenal nonmalignant tumor & 1 & Hernia & 2 \\
\hline Anal fistula & 1 & Autoimmune thyroiditis & 1 \\
\hline Calculus of kidney & 1 & Back pain & 1 \\
\hline Cerebral infarction & 1 & Cervical spondylosis & 1 \\
\hline Dental caries & I & Hypertension & I \\
\hline Diabetes mellitus & 1 & Osteoporosis & 1 \\
\hline Gastric ulcer & 1 & Phlebitis & I \\
\hline Low back pain & 1 & Premenstrual tension syndrome & 1 \\
\hline Lumbar spondylolisthesis & I & Post-surgery for lung cancer & 1 \\
\hline Osteoarthritis of knee & I & Pyogenic cervical discitis & I \\
\hline \multirow[t]{5}{*}{ Ulcerative colitis } & I & Rheumatoid arthritis & I \\
\hline & & Sprain of neck & I \\
\hline & & Systemic sclerosis & I \\
\hline & & Tension-type headache & 1 \\
\hline & & Varix of the lower extremity & I \\
\hline
\end{tabular}

Abbreviations: CR, complete remission; PR, partial remission; PPS, painful physical symptoms.

Neuropsychiatric Disease and Treatment

\section{Publish your work in this journal}

Neuropsychiatric Disease and Treatment is an international, peerreviewed journal of clinical therapeutics and pharmacology focusing on concise rapid reporting of clinical or pre-clinical studies on a range of neuropsychiatric and neurological disorders. This journa is indexed on PubMed Central, the 'PsycINFO' database and CAS,

\section{Dovepress}

and is the official journal of The International Neuropsychiatric Association (INA). The manuscript management system is completely online and includes a very quick and fair peer-review system, which is all easy to use. Visit http://www.dovepress.com/testimonials.php to read real quotes from published authors. 